\title{
Ultraperipheral vs. Ordinary Nuclear Interactions
}

\section{M. Dremin}

Lebedev Physical Institute, 119991 Moscow, Russia; dremin@lpi.ru

Received: 29 November 2019; Accepted: 23 December 2019; Published: 25 December 2019

Abstract: It is argued that the cross sections of ultraperipheral interactions of heavy nuclei can become comparable in value to those of their ordinary hadronic interactions at high energies. Simple estimates of corresponding "preasymptotic energy thresholds" are provided. The method of equivalent photons is compared with the perturbative approach. The situation at NICA/FAIR energies is discussed.

Keywords: proton; nuclei; ultraperipheral; cross section

\section{Introduction}

The cross sections of both ultraperipheral and ordinary hadronic nuclear interactions increase with the rise in collision energies. The rate of the increase is higher for ultraperipheral collisions with large impact parameters where the electromagnetic fields of colliding charged objects play a dominant role. Surely, at lower energies, their strength is weaker compared to effects due to strong hadronic (quark-gluon) interactions. Thus, starting from smaller initial values, ultraperipheral cross sections have the chance to overcome at higher energies the contribution of ordinary processes if the electromagnetic fields between colliding heavy nuclei are strong enough.

Landau and Lifshitz were the first to show [1] that the cross section $\sigma$ for the production of an electron and positron in ultraperipheral nuclear $(A)$ collisions increases with the cube of the logarithm of the energy $E$ :

$$
\sigma\left(A A \rightarrow A A e^{-} e^{+}\right) \propto \ln ^{3} \gamma,
$$

where $\gamma=E / m$ is the Lorentz boost ${ }^{1}$. It is important that the prefactor in Equation (1) is proportional to $Z^{4}$ where $Z$ is the nucleus charge. Therefore, the cross sections of ultraperipheral collisions of heavy nuclei are strongly enhanced. This result was obtained by considering the Dirac equation and new ideas of the creation of positrons from the Dirac sea.

In these collisions, the two colliding protons or nuclei interact electromagnetically but not hadronically. They effectively miss each other interacting by their photon clouds only, which create the electron-positron pairs. No nuclear transitions appear at small transferred momenta.

Such interactions were first considered by Fermi [2] almost a century ago. Ten years later, the method of equivalent photons $[1,3,4]$ was developed and effectively used for quantitative estimates. The photons in the clouds of fast moving nuclei can be considered almost real because their energy is much higher than their virtuality (the four-momenta squared). Unfortunately, this approximation is limited by asymptotic formulas, such as Equation (1). To get the preasymptotic behavior one should calculate the factor $\gamma_{0}$ in the ratio $\gamma / \gamma_{0}$ within the logarithm in Equation (1). The condition $\gamma \gg \gamma_{0}$ is necessary for asymptotics. To perform the program one has to use some knowledge of the structure of the colliding objects, the masses of produced particles etc. The new parameters enter the game.

Soon, the perturbative Born approximation was used by Racah [5] and preasymptotic terms with lower powers of $\ln \gamma$ were calculated using this approach. The main bulk of the total cross

1 The asymptotic dependence is not changed if the laboratory frame, used in Ref. [1], is replaced by the center of mass system. 
section is usually provided by hadronic interactions. The present experimental results from the energy behavior of the cross sections of proton-proton interactions displayed in Figure 1 [6,7] demonstrate the approximately linear (or slightly stronger) increase with the logarithm of the energy.

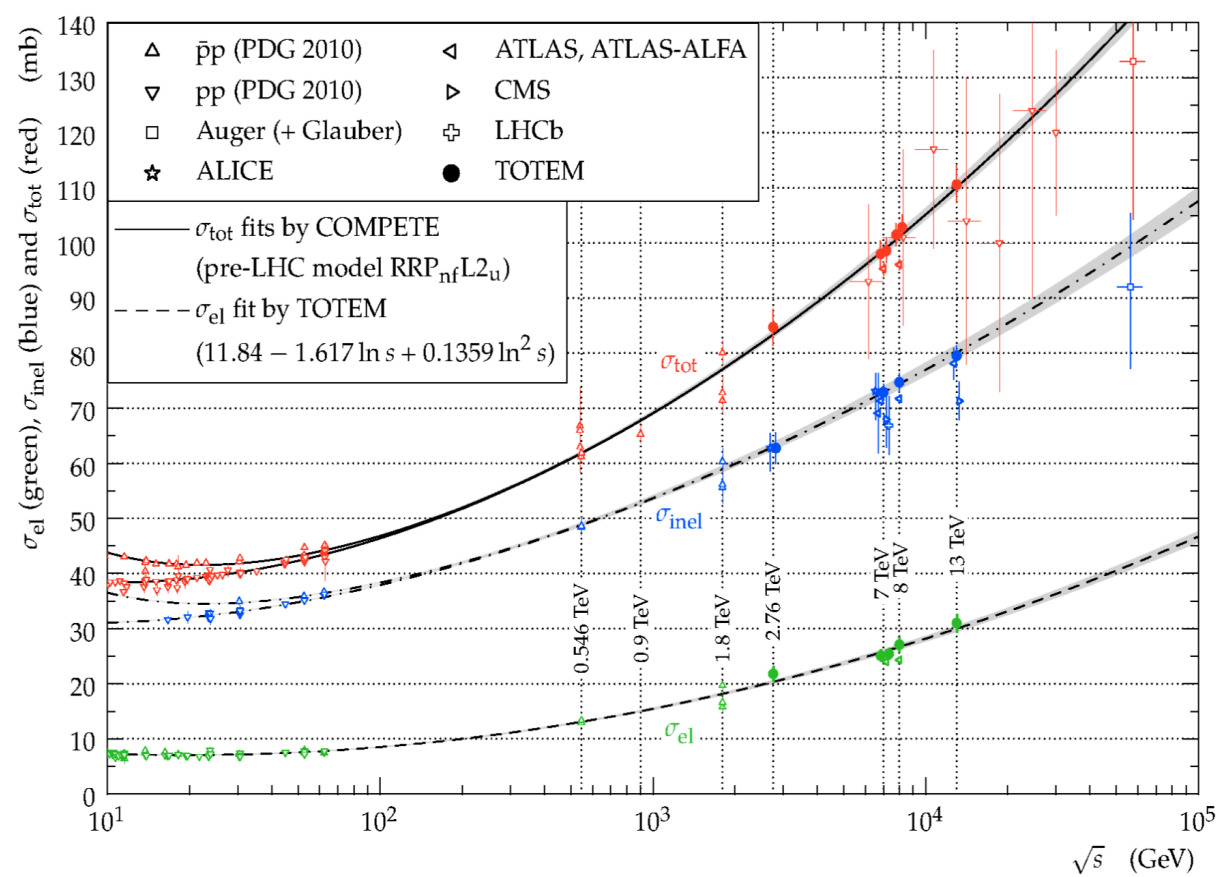

Figure 1. The energy dependence of the total, elastic and inelastic proton-proton cross sections.

The stronger regime, up to the square of the logarithm, is often used $[7,8]$ in practical fits. It is, in principle, admissible according to the famous Froissart bound [9] for purely hadronic interactions limited in space. Unfortunately, this theoretical bound is of no practical significance because it lies much above experimental results due to a quite large numerical factor in front of the logarithm squared.

The large spatial extention of electromagnetic forces, in its turn, leads to the stronger energy increase of some inelastic processes. In view of such competition of electromagnetic and strong forces it is desirable to estimate at which energies and other experimental conditions these two contributions become of a comparable size and, also, to show where the ultraperipheral processes start playing a role.

\section{Simple Preasymptotic Estimates}

The high density of photons in electromagnetic fields surrounding charged colliding objects is responsible for strong increase of ultraperipheral cross sections. The flux of photons is dominated by those carrying small fractions $x$ of the nucleon energy. The distribution of equivalent photons generated by a moving nucleus with the charge $\mathrm{Ze}$ (see, e.g., [10]) integrated over transverse momentum up to some value leads according to the method of equivalent photons to the flux

$$
\frac{d n}{d x}=\frac{2 Z^{2} \alpha}{\pi x} \ln \frac{u(Z)}{x}
$$

The ultraperipherality parameter $u(Z)$ depends on the nature of colliding objects and differs numerically in various approaches [11-17]. It has a physical meaning, which is the ratio of the maximum adoptable transverse momentum to the nucleon mass. It depends on charges $\mathrm{Ze}$, energy, sizes (formfactors) and impact parameters (the transverse distance between the centers) of colliding objects. The impact parameters cannot be measured but, surely, should exceed the sum of the radii. This requirement can be restated as a bound on the exchanged transverse momenta, such that the objects are not destroyed but slightly deflected by the collision so that no excitations or nuclear transitions 
happen. The bound depends on their internal structure, i.e., on forces inside them. These forces are stronger for a proton than for heavy nuclei. Therefore protons allow larger transverse momenta. The quantitative estimates of the parameter $u$ for different processes will be given below.

Besides the electron-positron pairs considered in Refs [1,5], other pairs of oppositely charged particles can be created in the two-photon collisions. For example, pairs of muons produced in ultraperipheral collisions are observed at LHC [18-20]. The light-by-light scattering described theoretically by the loop of charged particles is also detected at LHC [21]. Some neutral bosons composed of quark-antiquark pairs can be produced. This process is especially suitable for the compact theoretical demonstration [16] of $\ln ^{3} \gamma$-law (1). The exclusive cross section of the production of the resonance $R$ in collisions of nuclei $A$ can be written as

$$
\sigma_{A A}(R)=\int d x_{1} d x_{2} \frac{d n}{d x_{1}} \frac{d n}{d x_{2}} \sigma_{\gamma \gamma}(R),
$$

where the fluxes $d n / d x_{i}$ for the colliding objects 1 and 2 are given by Equation (2) and (see Ref. [11])

$$
\sigma_{\gamma \gamma}(R)=\frac{8 \pi^{2} \Gamma_{t o t}(R)}{m_{R}} B r(R \rightarrow \gamma \gamma) B r_{d}(R) \delta\left(x_{1} x_{2} s_{n n}-m_{R}^{2}\right)
$$

Here $m_{R}$ is the mass of $R, \Gamma_{\text {tot }}(R)$ its total width and $B r_{d}(R)$ denotes the branching ratio to a considered channel of its decay. $s_{n n}=(2 m \gamma)^{2}, m$ is a nucleon mass. The $\delta$-function approximation is used for resonances with small widths compared to their masses.

The integrals in Equation (3) can be easily calculated so that one gets the analytical formula

$$
\sigma_{A A}(R)=\frac{128}{3} Z^{4} \alpha^{2} B r(R \rightarrow \gamma \gamma) B r_{d}(R) \frac{\Gamma_{t o t}(R)}{m_{R}^{3}} \ln ^{3} \frac{2 u m \gamma}{m_{R}}
$$

The factor $2 m u / m_{R}=1 / \gamma_{0}$ defines the preasymptotic behavior of the ultraperipheral cross section of production of the resonance $R$.

It can be confronted with the formula for ultraperipheral production of muon pairs in protonproton collisions derived in Equation (7) of [17]:

$$
\sigma\left(p p(\gamma \gamma) \rightarrow p p \mu^{+} \mu^{-}\right)=8 \frac{28}{27} \frac{\alpha^{4}}{\pi m_{\mu}^{2}} \ln ^{3} \frac{u m \gamma}{m_{\mu}} .
$$

The energy dependence of both processes is the same for $m_{R}=2 m_{\mu}$ as expected. The preasymptotic behavior is determined by the factor $u m / m_{R}$. The asymptotic limit is reached at

$$
\gamma \gg m_{R} / 2 u m
$$

where the terms increasing slower than $\ln ^{3} \gamma$ can be neglected. The parameter $u$ is the least precisely determined element of the whole approach. The careful treatment of formfactors of protons and nuclei with account of the photon virtuality (see also Refs. $[12,14]$ where the problem was treated in more detail) and the suppression factors [17] lead to its values $u_{p p} \approx 0.2$ for $p p$ and $u_{P b P b} \approx 0.02$ for $\mathrm{PbPb}$-collisions within the factors about 1.5 which depend on the particular shape of the formfactors (see [17]). In what follows, motivated by these results I use the parameters $u$ obtained in Ref. [17]. They favor ultraperipheral processes at lower energies than the extremely strong ad-hoc requirements of the cutoff of the impact parameters imposed in [15] and used in [16] which give rise to about 4 times smaller values of $u$, i.e., to the higher lying (on the energy scale) asymptotics.

Taking into account these caveats, one can confront the values and energy dependences of experimentally measured cross sections of inelastic $p p$-interactions and the relative contribution of 
ultraperipheral processes in them. The values of the inelastic cross section shown in Figure 1 are rather well approximated in the energy interval from $60 \mathrm{GeV}$ to $13 \mathrm{TeV}$ by the expression

$$
\sigma_{\text {inel }}(s)=8.2 \ln (1.37 \sqrt{s}) m b,
$$

where $\sqrt{s}$ is in $\mathrm{GeV}$.

Let us consider the channel with a single $\pi^{0}$ produced among all inelastic channels and compare it with the expression for the ultraperipheral cross section for $\pi^{0}$-production. The multiplicity distribution is well described in the considered energy interval by a composition of the negative binomial distributions (NBD) [22] with the average multiplicity $\bar{n}$ and the dispersion determined by $k$. It is dominated by a single NBD for events with low multiplicities. The probability to get the inelastic process with a charged pion produced is equal to

$$
P\left(\pi^{ \pm}\right)=\bar{n}\left(1+\frac{\bar{n}}{k}\right)^{-k-1}
$$

It is twice smaller for a neutral pion, such that $P\left(\pi^{0}\right) \approx 4 \times 10^{-3}$ for $\bar{n}=13, k=4.4$ at the intermediate (for the chosen interval) energy $1.8 \mathrm{TeV}$ (see [22]). The product $P\left(\pi^{0}\right) \sigma_{\text {inel }}(s)$ must be compared with Equation $(5)^{2}$ for $Z=1$. The preasymptotic factors in logarithms are very close to one another (1.37 in (8) compared to 1.48 in (5) for $u$ chosen according to Ref. [17]). Thus, the cubic equation obtained from the equality of the product $P\left(\pi^{0}\right) \sigma_{\text {inel }}(s)$ to Equation (5) reduces approximately to the quadratic one. The energy $s_{0}$, at which the cross section of the ultraperipheral production of a single $\pi^{0}$ becomes equal to its partial cross section due to hadronic interactions, can be estimated from the equality

$$
2.7 \times 10^{-9} \ln ^{2} \sqrt{s_{0}} \approx 3.28 \times 10^{-2} .
$$

The photon fluxes for $p p$ collisions with $Z=1$ are not strong (see Equations (2) and (3)). Therefore, the factor in front of the ultraperipheral contribution on the left hand side is extremely small. One concludes that these expressions can become equal only at the unrealistically high energy $\sqrt{s_{0}} \approx e^{3500} \mathrm{GeV}$. At first sight, it seems hopeless to measure such processes at the present energies in $p p$-collisions. To enlarge their share, one should try to impose some special experimental cutoffs. Fortunately, there are distinctive features which can help in choices of such events. In particular, the ultraperipherally created neutral pions move slowly, decay to two photons with energies $67.5 \mathrm{MeV}$ and are strongly concentrated near central rapidity. The whole process looks like the light-by-light scattering at the specific $\pi^{0}$ energy. Surely, the fiducial cross sections of both ultraperipheral and hadronic interactions would be strongly diminished.

The optimism is supported by studies [17] of ultraperipheral production of $\mu^{+} \mu^{-}$pairs. The ultraperipheral cross section (6) at $13 \mathrm{TeV}$ is equal ${ }^{3}$ to $0.22 \mu \mathrm{b}$. It is much smaller than the inelastic cross section of $80 \mathrm{mb}$. Further cuts on the invariant mass of the $\mu^{+} \mu^{-}$pair, on the muon transverse momentum and pseudorapidity reduce its value to $3.35 \mathrm{pb}$. If corrected for absorptive effects [24] it gives $3.06 \pm 0.05 \mathrm{pb}$. The chosen cuts coincide with those imposed in studies of the ATLAS collaboration [18] which lead to the value $3.12 \pm 0.07$ (stat.) \pm 0.10 (syst.) pb. The Monte Carlo program [25] which incorporates both ordinary and ultraperipheral processes predicts $3.45 \pm 0.06 \mathrm{pb}$. Theoretical results are in agreement with experimental data and show that ultraperipheral processes dominate over other sources in this fiducial volume. Analogous conclusions were obtained for lead-lead collisions [17]. The measured fiducial cross sections are on the $\mu \mathrm{b}$ scale compared to $\mathrm{pb}$ 's for $p p$.

The $\pi^{0}$-production in $2 \gamma$-collisions was originally suggested by Low [23].

Please note that it includes the $\ln ^{3} \gamma$-factor which is about 700 . 
The creation of a $\pi^{0}$ in collisions of heavy nuclei is strongly enhanced by the factors $Z^{4}=4.5 \times 10^{7}$ for $\mathrm{PbPb}$ or $3.9 \times 10^{7}$ for $\mathrm{AuAu}$ collisions which must appear on the left hand side of the equation analogous to (10). That makes it of comparable size to the hadronic contribution on the right hand side even if the larger nuclear cross sections (of the order of the geometrical size about $1500 \mathrm{mb}$ ) are inserted there. The factor of the stronger energy increase of ultraperipheral processes becomes decisive now. Taking into account the value of $u \approx 0.02$ applicable to heavy nuclei, the effect could become observable even at comparatively low energies of NICA (with $\gamma=4.5-6$ ) because the preasymptotic threshold (7) ${ }^{4}$ asks for $\gamma>3.6$. Again, photons with energies $67.5 \mathrm{MeV}$ in the central rapidity region can be looked for as a signature for decays of slowly moving neutral pions produced in ultraperipheral collisions. The threshold for heavier resonances is proportional to their masses (see (7)) and, therefore, moves to higher energies. Quantitative comparison can be done after the Monte Carlo program for the exclusive resonance production similar to the STARlight program for $\mu^{+} \mu^{-}$processes [26] is elaborated and helps in search of the proper fiducial phase space volume.

Special attention should be paid at NICA to the production of $e^{+} e^{-}$-pairs ${ }^{5}$. If observed, it would show that ultraperipheral processes survive even at comparatively low NICA energies. Their cross section can be estimated according to the Racah formula $[5,14]$ which accounts for the terms that increase slower with energy in the perturbative Born approximation:

$$
\sigma=\frac{28}{27 \pi} \frac{Z^{4} \alpha^{4}}{m_{e}^{2}}\left[L^{3}-2.2 L^{2}+3.84 L-1.64\right],
$$

where $m_{e}$ is the electron mass, $L=\ln \gamma^{2}$. For the energy $4.5 \mathrm{GeV}$ per nucleon in $A u A u$ collisions one gets very large cross section $\sigma \approx 1 \mathrm{~kb}$ exceeding the values for geometrical estimates of hadronic processes at low impact parameters. The small electron mass plays a crucial role. The $L^{2}$ term is negative and about $30 \%$ of the main $L^{3}$-term which dominates asymptotically. Even at the LHC energy $5 \mathrm{TeV}$ it is about $10 \%$. Moreover, the $L^{2}$-term of the Coulomb corrections to the Racah formula is also negative [28-30]

$$
\sigma_{C}=-\frac{56}{9 \pi} \frac{Z^{4} \alpha^{4}}{m_{e}^{2}} f(Z) L^{2}
$$

where

$$
f(Z)=(Z \alpha)^{2} \sum_{n=1}^{\infty} \frac{1}{n\left(n^{2}+(Z \alpha)^{2}\right)} .
$$

These corrections are large at NICA energies. Effectively, they can be accounted by the replacement of the coefficient -2.2 in front of the $L^{2}$ term by -4.2 . The preasymptotic "threshold" determined by the requirement that $L^{3}$-contribution is larger than that of the whole $L^{2}$-term is shifted to higher energies and poses a problem at NICA.

However, for quantitative treatment, the proper account of the nuclei structure should be incorporated phenomenologically in these perturbative calculations. The nuclei were treated in Refs. $[1,5]$ as pointlike objects. The strong dependence of preasymptopia on their structure (the parameter $u$ ) and on masses of created particles is claimed according to the method of equivalent photons. If Equation (5) is used for estimates of the $L^{2}$-term with $m_{R}=2 m_{e}$ one finds quite large positive coefficients of values about 10 in front of it. That would compensate the negative contributions discussed above and shift the "threshold" to lower energies. In any case, the estimated values of the cross section are still quite optimistic. Equation (11) cannot be trusted at $\gamma$ approaching 3 where the second term becomes equal to the first one. The background from collisions at low impact parameters at NICA must be much

4 The previous estimate of the preasymptotic threshold [16] was 4 times larger as mentioned above and excluded NICA energies. 5 Let us mention that at the LHC this process was studied in $\mathrm{PbPb}$ collisions at $\sqrt{s}=2.76 \mathrm{TeV}$ by the ALICE collaboration [27]. 
smaller than at LHC energies. Surely, special detectors must be installed to separate electrons from other products of the collisions.

The muon pairs cannot be created ultraperipherally at NICA energies. The corresponding preasymptotic factor $u m / m_{\mu}$ in Equation (6) becomes about 200 times smaller than $u m / m_{e}$ for the $e^{+} e^{-}$-case. In other words, it means that the parameter $\gamma_{0}$ becomes 200 times larger. That leads to the negative $L^{2}$-term, i.e., to a higher "threshold".

Let me mention at the very end that the planned NICA energies are very close to those at the FAIR facilities. Thus, all conclusions concerning the ultraperipheral processes are equivalent in both cases.

\section{Conclusions}

Electromagnetic fields of colliding charged particles are the reason for the fast increase of their ultraperipheral cross sections with energy. These cross sections are strongly enlarged by the nuclear charge $Z$ for interactions of heavy ions. The exclusive production of resonances in these processes is compared with cross sections of ordinary hadronic interactions for $p p$ and $P b P b$ high energy collisions. It is small in $p p$ and quite noticeable in $\mathrm{PbPb}$ processes due to the $Z^{4}$-factor. Using the parameters of preasymptotic estimates borrowed from [17], it is argued that the ultraperipheral processes $A A \rightarrow A A \pi^{0}$ and $A A \rightarrow A A e^{+} e^{-}$can be observed at pernucleon energies higher than $3.6 \mathrm{GeV}$.

Funding: This work was supported by the RFBR project 18-02-40131 and RAN-CERN program.

Acknowledgments: I am grateful to C. Bertulani for references [12,14].

Conflicts of Interest: The author declares no conflict of interest.

\section{References}

1. Landau, L.D.; Lifshitz, E.M. On the production of electrons and positrons by a collision of two particles. Physikalische Zeitschrift der Sowjetunion 1934, 6, 244.

2. Fermi, E. On the Theory of the impact between atoms and electrically charged particles. Zeitschrift Für Physik 1924, 29, 315-327. [CrossRef]

3. Weizsäcker, C.F.V. Radiation emitted in collisions of very fast electrons. Zeitschrift Für Physik 1934, 88, 612-625. [CrossRef]

4. Williams, E.J. Nature of the high energy particles of penetrating radiation and status of ionization and radiation formulae. Phys. Rev. 1934, 45, 729-730. [CrossRef]

5. Racah, G. Sulla nascita di coppie per urti di particelle elettrizzate. Nuovo Cim. 1937, 14, 93. [CrossRef]

6. Aspell, P.; Atanassov, I.; Avati, V.; Baechler, J.; Barrera, C.B.; Berardi, V.; Berretti, M.; Bossini, E.; Bottigli, U.; Bozzo, M.; et al. [The TOTEM Collaboration] First measurement of elastic, inelastic and total cross-section at $\sqrt{s}=13 \mathrm{TeV}$ by TOTEM and overview of cross-section data at LHC energies. arXiv 2017, arXiv:1712.06153.

7. Dremin, I.M. Some Recent Results on High-Energy Proton Interactions. Particles 2019, 2, 57-69. [CrossRef]

8. Royon, C. Total cross section and particle production in soft and hard processes at the LHC. arXiv 2019, arXiv:1909.12696.

9. Froissart, M. Asymptotic behavior and subtractions in the Mandelstam representation. Phys. Rev. 1961, 123, 1053-1057. [CrossRef]

10. Berestetsky, V.B.; Lifshitz, E.M.; Pitaevsky, L.P. Kvantovaya Electrodinamika; Fizmatlit: Moscow, Russia, 2001.

11. Budnev, V.M.; Ginzburg, I.F.; Meledin, G.V.; Serbo, V.G. The two-photon particle production mechanism. Physical problems. Applications. Equivalent photon approximation. Phys. Rep. C 1975, 15, 181-282. [CrossRef]

12. Baur, G.; Bertulani, C.A. $\gamma-\gamma$ physics with peripheral relativistic heavy ion collisions. Z. Phys. A 1988, 330, 77-81.

13. Klein, S.R.; Nystrand, J. Interference in exclusive vector meson production in heavy-ion collisions. Phys. Rev. Lett. 2000, 84, 2330-2333. [CrossRef] [PubMed]

14. Bertulani, C.A.; Klein, S.R.; Nystrand, J. Physics of ultra-peripheral nuclear collisions. Ann. Rev. Nucl. Part. Sci. 2005, 55, 271-310. [CrossRef] 
15. Khoze, V.A.; Martin, A.D.; Ryskin, M.G. Exclusive vector meson production in heavy ion collisions. arXiv 2019, arXiv:1902.08136.

16. Dremin, I.M. Geometry of ultraperipheral nuclear collisions. Int. J. Mod. Phys. A 2019, 34, 1950068. [CrossRef]

17. Vysotsky, M.I.; Zhemchugov, E.V. Equivalent photons in proton-proton and ion-ion collisions at the Large Hadron Collider. Phys. Usp. 2019, 189, 975-984. [CrossRef]

18. Aaboud, M. [ATLAS Collaboration] Measurement of the exclusive gamma gamma-> mu (+) mu (-) process in proton-proton collisions at root $\mathrm{s}=13 \mathrm{TeV}$ with the ATLAS detector. Phys. Lett. B 2018, 777, 303-323. [CrossRef]

19. Aaboud, M. [ATLAS collaboration] Expected performance of the ATLAS inner tracker at the High-Luminosity LHC. ATLAS-CONF-2016-025. In Proceedings of the 3rd ECFA High Luminosity LHC Experiments Workshop, Aix-Les-Bains, France, 3-6 October 2016.

20. Arratia, M. Ultra-peripheral Collisions with the ATLAS Detector. arXiv 2016, arXiv:1611.05145.

21. d'Enterria, D. [CMS Collaboration] Evidence for light-by-light scattering in ultraperipheral $\mathrm{PbPb}$ collisions at $\sqrt{s N N}=5.02$ TeV. Nucl. Phys. A 2019, 982, 791-794. [CrossRef]

22. Dremin, I.M.; Nechitailo, V.A. Soft multiple parton interactions as seen in multiplicity distributions at Tevatron and LHC. Phys. Rev. D 2011, 84, 034026. [CrossRef]

23. Low, F.E. Proposal for Measuring the $\pi^{0}$ Lifetime by $\pi^{0}$ Production in Electron-Electron or Electron-Positron Collisions. Phys. Rev. 1960, 120, 582-583. [CrossRef]

24. Dyndal, M.; Schoeffel, L. The role of finite-size effects on the spectrum of equivalent photons in proton-proton collisions at the LHC. Phys. Lett. B 2015, 741, 66-70. [CrossRef]

25. Harland-Lang, L.A.; Khoze, V.A.; Ryskin, M.G. Exclusive physics at the LHC with SuperChic 2. Eur. Phys. J. 2016, 76, 9. [CrossRef]

26. Klein, S.R.; Nystrand, J.; Seger, J.; Gorbunov, Y.; Butterworth, J. STARlight: A Monte Carlo simulation program for ultra-peripheral collisions of relativistic ions. Comput. Phys. Commun. 2017, 212, 258-268. [CrossRef]

27. Abbas, E. [The ALICE Collaboration] Charmonium and e+ e- pair photoproduction at mid-rapidity in ultra-peripheral $\mathrm{Pb}-\mathrm{Pb}$ collisions at $\sqrt{s N N}=2.76 \mathrm{TeV}$. Eur. Phys. J. 2013, 73, 2617. [CrossRef]

28. Ivanov, D.Y.; Schiller, A.; Serbo, V.G. Large Coulomb corrections to the e+ e- pair production at relativistic heavy ion colliders. Phys. Lett. B 1999, 454, 155-160. [CrossRef]

29. Lee, R.N.; Milstein, A.I.; Serbo, V.G. Structure of the Coulomb and unitarity corrections to the cross section of e+ e- pair production in ultrarelativistic nuclear collisions. Phys. Rev. A 2002, 65, 022102. [CrossRef]

30. Gevorkyan, S.R.; Kuraev, E.A. Lepton pair production in relativistic ion collisions to all orders in $\mathrm{Z} \alpha$ with logarithmic accuracy. J. Phys. G 2003, 29, 1227-1236. [CrossRef]

(C) 2019 by the authors. Licensee MDPI, Basel, Switzerland. This article is an open access article distributed under the terms and conditions of the Creative Commons Attribution (CC BY) license (http://creativecommons.org/licenses/by/4.0/). 